\title{
Genome-wide characterization and expression analysis of the HD-Zip gene family in response to drought and salinity stresses in sesame
}

\author{
Mengyuan Wei ${ }^{1}$, Aili Liu', Yujuan Zhang ${ }^{1,2}$, Yong Zhou ${ }^{3}$, Donghua Li ${ }^{1}$, Komivi Dossa ${ }^{1}$, Rong Zhou'
} Xiurong Zhang ${ }^{1 *}$ and Jun You ${ }^{1 *}$ (D)

\begin{abstract}
Background: The homeodomain-leucine zipper (HD-Zip) gene family is one of the plant-specific transcription factor families, involved in plant development, growth, and in the response to diverse stresses. However, comprehensive analysis of the HD-Zip genes, especially those involved in response to drought and salinity stresses is lacking in sesame (Sesamum indicum L.), an important oil crop in tropical and subtropical areas.

Results: In this study, 45 HD-Zip genes were identified in sesame, and denominated as SiHDZ01-SiHDZ45. Members of SiHDZ family were classified into four groups (HD-Zip I-IV) based on the phylogenetic relationship of Arabidopsis HDZip proteins, which was further supported by the analysis of their conserved motifs and gene structures. Expression analyses of SiHDZ genes based on transcriptome data showed that the expression patterns of these genes were varied in different tissues. Additionally, we showed that at least $75 \%$ of the SiHDZ genes were differentially expressed in responses to drought and salinity treatments, and highlighted the important role of HD-Zip I and II genes in stress responses in sesame.
\end{abstract}

Conclusions: This study provides important information for functional characterization of stress-responsive HD-Zip genes and may contribute to the better understanding of the molecular basis of stress tolerance in sesame.

Keywords: Sesame, Transcription factors, HD-ZIP, Abiotic stress, Gene expression

\section{Background}

Transcription factors (TFs) play a vital role in regulatory networks that link between the developmental program and response of genes to endogenous and environmental signals. TFs directly bind to the promoters of target genes in a sequence-specific manner to activate or repress the expression of their target genes [1]. Homeobox proteins are a large superfamily of transcription factors (TFs) found in invertebrates, vertebrates, fungi, and plants, which contain a highly conserved DNA-binding domain known as the homeodomain (HD) [2]. The HD is 60 amino acids in length and adopts a structure of

\footnotetext{
* Correspondence: zhangxr@oilcrops.cn; junyou@caas.cn

${ }^{1}$ Key Laboratory of Biology and Genetic Improvement of Oil Crops, Ministry of Agriculture and Rural Affairs, Oil Crops Research Institute of the Chinese Academy of Agricultural Sciences, Wuhan 430062, China

Full list of author information is available at the end of the article
}

three $\alpha$-helices connected by a loop and a turn [2-4]. The HD binds DNA as a monomer with high affinity through the interactions established by helix III (called the recognition helix) with the major groove of the target DNA while the disordered N-terminal arm, located upstream the first helix, interacts with the DNA minor groove [5]. Homeodomain proteins have been classified into different families according to the distinguishing features, such as HD location, association with other domains, their size and gene structures. Based on thorough analyses of homeodomain proteins from flowering plants, moss, Selaginella, unicellular green algae, and red algae, Mukherjee et al. [6] classified the plant HDcontaining proteins into 14 families, including HD-Zip (homeodomain associated to a leucine zipper), WOX

(c) The Author(s). 2019 Open Access This article is distributed under the terms of the Creative Commons Attribution 4.0 International License (http://creativecommons.org/licenses/by/4.0/), which permits unrestricted use, distribution, and reproduction in any medium, provided you give appropriate credit to the original author(s) and the source, provide a link to the Creative Commons license, and indicate if changes were made. The Creative Commons Public Domain Dedication waiver (http://creativecommons.org/publicdomain/zero/1.0/) applies to the data made available in this article, unless otherwise stated. 
(Wuschel related homeobox) and KNOX (Knotted related homeobox).

The plant-specific HD-Zip transcription factors contain a leucine zipper motif (LZ) that acts as a dimerization motif, located in the carboxyl terminal to the homeodomain $[2,6]$. The HD-ZIP family further divided into four subfamilies, HD-Zip I-IV, according to the conserved HDZip domain, additional conserved domain, structure features and functions [6]. Members of HD-Zip I and II family share the conserved HD and LZ domains, and bind similar pseudo-palindromic cis-elements, CAATNATTG, where $\mathrm{N}$ can be $\mathrm{A} / \mathrm{T}$ or $\mathrm{G} / \mathrm{C}$ for HD-Zip I or II homeodomains, respectively $[7,8]$. Besides HD and LZ domains, HD-ZIP II proteins present a conserved CPSCE motif (named by five conserved amino acids: Cys, Pro, Ser, Cys and Glu) downstream of the LZ [9]. Both the HD-Zip III and HD-Zip IV subfamily proteins are characterized by the START (steroidogenic acute regulatory protein-related lipid transfer) domain and START adjacent domain (SAD) $[6,10]$. HDZip III proteins can be distinguished from HD-Zip IV proteins by the presence of C-terminal MEKHLA domain which is absent in the HD-ZIP IV proteins [11]. HD-Zip III proteins bind the consensus sequence GTAAT [G/C] ATTAC, while members of HD-Zip IV family recognized the sequence TAAATG $[\mathrm{C} / \mathrm{T}]$ A $[12,13]$.

There are 48 and 49 HD-ZIP genes in Arabidopsis thaliana and rice (Oryza sativa L.), respectively [6, 14, 15]. Through genome-wide analysis, members of HD-Zip gene family have been also identified in many other plant species, including grape (Vitis vinifera) [16], maize (Zea mays) [17], soybean (Glycine max) [18, 19], cassava (Manihot esculenta) [20], wheat (Triticum aestivum) [21], tea plant (Camellia sinensis) [22], and potato (Solanum tuberosum) [23]. HD-Zip proteins are known to participate in transcriptional regulation of various biological processes, and members of the different subfamilies have specific roles $[2$, 24, 25]. HD-Zip I proteins were found to be implicated in the regulation of abiotic stress responses, light and hormone (ABA, auxin and ethylene) signal transduction, and plant growth and development [2, 24, 26]. AtHB7 (Arabidopsis thaliana HOMEOBOX 7) and AtHB12 (Arabidopsis thaliana HOMEOBOX 12) from Arabidopsis HD-Zip I group participate in $\mathrm{ABA}$ sensing and transduction, playing a key role in drought and salt responses [27, 28]. MtHB1 (MEDICAGO TRUNCATULA HOMEOBOX 1), a HD-Zip I protein from $M$. truncatula, regulates root architecture under adverse environmental stresses by repressing LBD1 (LOB-BINDING DOMAIN 1) involving crosstalk between auxin and ABA signaling pathways [29]. In the case of HD-Zip II proteins, they are mainly involved in development, shade avoidance and abiotic stress responses [3032]. For example, members of the HD-Zip II family, including AtHB2 (Arabidopsis thaliana HOMEOBOX 2), AtHB4 (Arabidopsis thaliana HOMEOBOX 4), and
HAT3 (HOMEOBOX FROM Arabidopsis thaliana 3), play crucial roles in regulation of leaf polarity and shade avoidance response [31, 33]. Two other HD-Zip II proteins, AtHB17 (Arabidopsis thaliana HOMEOBOX 17) and ABIG1 (ABA INSENSITIVE GROWTH 1), are involved in ABA-mediated stress response or growth inhibition [34, 35]. HD-Zip III proteins were reported to be involved in apical meristem formation, vascular development, organ polarity establishment, as well as auxin biosynthesis, transport and response $[31,36]$. Rice LF1 (LATERAL FLORET 1) gene, encoding a class III HD-ZIP protein, induced the three-florets spikelet by directly regulating the expression of meristem maintenance gene OSH1 (ORYZA sativa $H O M E O B O X$ 1) [37]. HD-Zip IV proteins play critical role in the specification of the protoderm, anthocyanin accumulation, and environmental responses [38]. For example, GhHOX3 (GOSSYPIUM HIRSUTUM HOMEOBOX 3) in this subfamily plays a central role in controlling cotton fibre elongation [39].

Sesame is an ancient and important oil crop, which is grown mainly in tropical and subtropical areas of the world. Sesame has been widely used in baked and confectionery products and edible oil due to its highly stable oil and high quantities of nutritious amino acids, minerals, vitamins, and lignans [40]. However, sesame production and quality is threatened by drought, salinity and other environmental stresses [41-43]. A series of TFs, such as ERF, WRKY, MYB, NAC and bZIP, have been genome-wide analyzed in sesame, and some stressresponsive TFs have been identified [44-48]. However, response to abiotic stress of HD-Zip genes was unclear in sesame. In this study, we systematically characterized the HD-Zip gene family in sesame, and analyzed their phylogenetic relationships, conserved motifs and gene structure, as well as expression patterns in different tissues and in response to abiotic stresses. Our results provide a perspective for further investigation of the functions of stress-responsive HD-ZIPs in sesame.

\section{Results \\ Genome-wide identification of HD-zip family genes in sesame}

For the genome-wide identification of HD-Zip (homeodomain-leucine zipper) family genes in sesame, the Hidden Markov Model (HMM) profile of the homeodomain (HD) (PF00046) and the leucine zipper (LZ) domain (PF02183) were employed as queries to search against the Sinbase database (http://ocri-genomics.org/Sinbase) using the program HMM3.0. In addition, the known HD-Zip protein sequences from Arabidopsis were obtained from the TAIR database based on a previous study [2], and these sequences were also used as queries for searches in the Sinbase database. After removing redundant sequences, the SMART database was used to 
examine the presence of the HD and LZ domains for each identified candidate. As a result, a total of $45 \mathrm{HD}$ Zip genes were identified in sesame, and they were designated as SiHDZ1-SiHDZ45 according to their chromosomal locations on the sesame linkage groups (LGs). The identified sesame SiHDZ genes encoded proteins ranging from a minimum of 160 (SiHDZ37) to a maximum of 847 (SiHDZ22) amino acids in length. Detail information of SiHDZs such as gene locus ID, linkage group location, proteins length, and other corresponding information are shown in Additional file 2: Table S1.

\section{Chromosomal localization and gene duplication analysis of SiHDZ genes}

The chromosomal localization of SiHDZ genes was determined to visualize their genomic position information (Fig. 1). Of the $45 \mathrm{SiHDZ}$ genes, 44 genes were distributed unequally on 12 out of the 16 LGs, with the LG08 having the majority of SiHDZ genes (7), whereas the LG07 had only one gene. In addition, one SiHDZ gene (SiHDZ45) was mapped to the unanchored scaffold, and is not shown in Fig. 1.

To study the potential gene duplication within the sesame genome, we examined the segmental and tandem duplication events in SiHDZ gene family during evolution. No tandem duplication events were found, while 23 gene pairs involving $36 \mathrm{SiHDZ}$ genes resulted from segment duplication (Additional file 1: Figure S1).

\section{Phylogenetic analysis amongst the Arabidopsis and sesame HDZs}

To reveal the phylogenetic relationships among the SiHDZ proteins, an unrooted phylogenetic tree was created to assess the genetic relationships between Arabidopsis and sesame HDZs. As shown in Fig. 2, these proteins can be divided into four distinct groups (HD-Zip I-IV), which is similar to that described in previous studies $[2,16]$. The numbers of HD-Zip I-IV members in sesame were 16, 10, 9, and 10, respectively (Additional file 2: Table S1; Fig. 2). The results provide an important basis for functional prediction of HD-Zip proteins in sesame.

\section{Gene structure and conserved domain analysis of SiHDZ genes}

To investigate the structural diversity of SiHDZ genes, Gene Structure Display Server (http://gsds.cbi.pku.edu.cn/ index.php) was employed to analyze the exon-intron structures of SiHDZ genes by comparing their coding sequences and corresponding genomic sequences. The results showed that the number of introns of SiHDZ genes varied between 0 and 17, but the genes that clustered together had similar number of introns (Fig. 3a, b). For example, all of the SiHDZ genes in group III contained the largest number of introns (17), members of $S i H D Z$ group IV had 8-10 introns, with the exception of SiHDZ17, which contained 7 introns (Fig. 3b). Compared to group III and IV, SiHDZ genes in group I and II had much fewer introns. SiHDZ genes in group II usually contain $2-3$ introns, whereas SiHDZ genes in group I feature 1-2

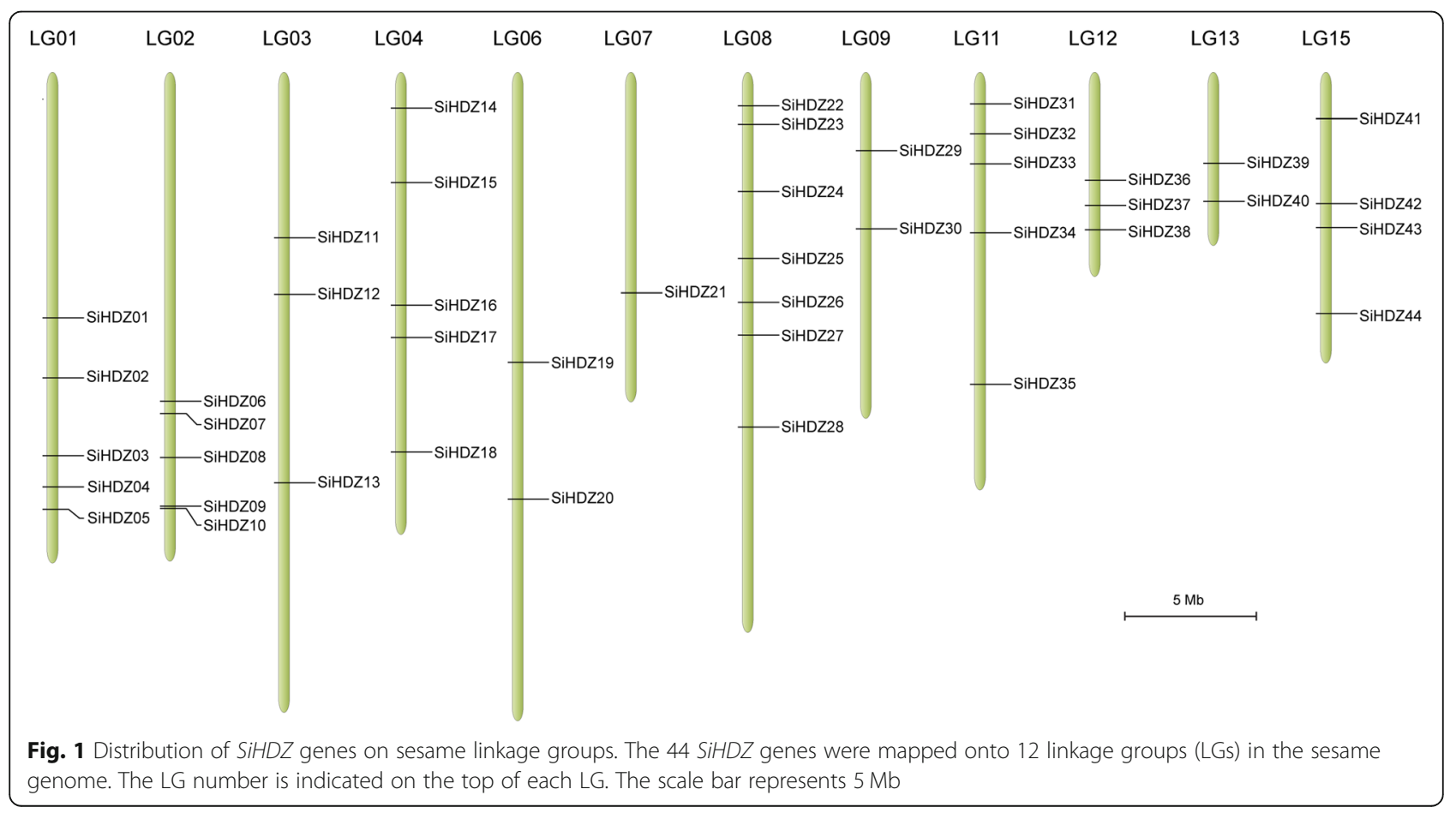




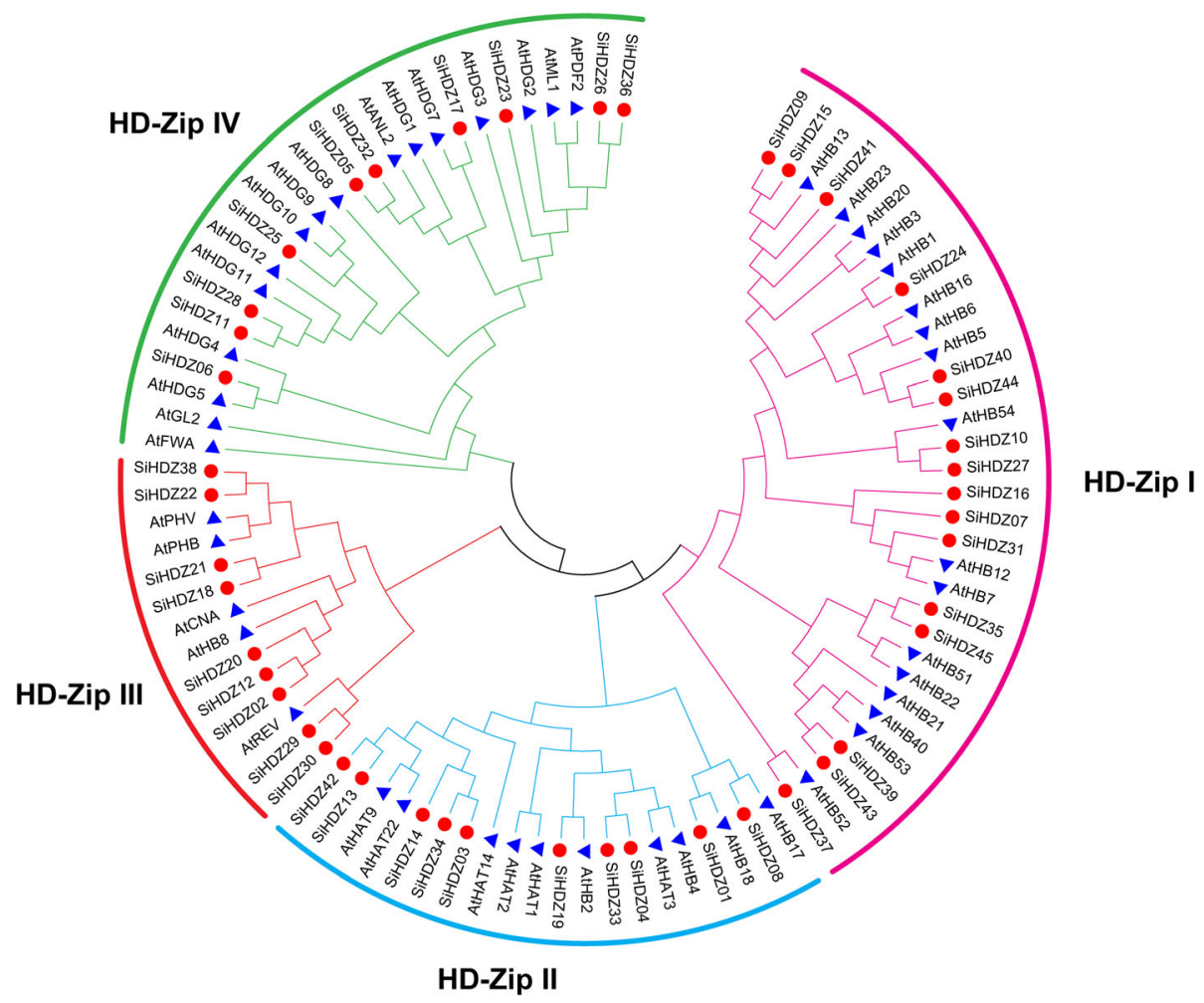

Fig. 2 Phylogenetic analysis of HD-Zip proteins from sesame and Arabidopsis. The sequences were aligned by Clustal X and the tree was generated using the NJ method in MEGA 5.0 with 1000 bootstrap replicates. The blue triangles and red dots represent HD-Zip proteins in Arabidopsis and sesame, respectively

introns, except for SiHDZ24 and SiHDZ37, whose number of introns were 3 and 0 , respectively (Fig. 3b).

To further study the origin and evolutionary pattern of SiHDZs, their protein sequences were subjected to the MEME tool and a total of 20 conserved motifs were identified. The identified motifs ranged from 24 to 50 amino acids in length. The details of the sequence logo of each motif were presented in Additional file 1: Figure S2. Amongst them, motif 1 and 2, corresponding to the homeobox domain, and motif 5 , corresponding to the LZ domain, were in common among all of the SiHDZs (Fig. 3c). In general, members in the same group harbored the similar motif organizations, while the motifs were divergent among different groups. For example, motifs $3,4,8$, and 12, which correspond to START domain, were shared in group III and group IV, while motif 9 and 16, which correspond to MEKHLA domain, were present in group III but absent in group IV. In addition, motif 9 was exclusively present in group II, motif 13 and 19 only existed in group III, while motifs 10 and 17 was only present in the group IV HDZs (Fig. $3 c$ ). These group-specific motifs may imply diverse functions of the HDZ family in sesame.

\section{Expression profiles of SiHDZ genes in different tissues}

To study the potential functions of the SiHDZ genes, we analyzed the expression profiles of SiHDZ genes in different tissues, including root, stem, flower, leaf, capsule and seed, based on the transcriptome data from SesameFG database [49]. As shown in Fig. 4, 25 SiHDZ genes were commonly detected (TPM value $>1$ ) in all of the tissues, suggesting that these genes might play a universal role in the tested tissues. Among them, four SiHDZ genes (SiHDZO4, 27, 29, and 40) were highly expressed (TPM value $>10$ ) in all six tissues, while SiHDZO3 and SiHDZ19 were most highly expressed (TPM value >90) in root. In addition, some SiHDZ genes were shown to have tissue-specific expression (Fig. 4). For example, SiHDZ11, 17, 28 and 38 displayed relatively low expression levels in root and capsule, but high expression in stem, flower, leaf and seed. SiHDZ31 and SiHDZ43 exhibited low expression levels in leaf, but relatively high expression levels in other tissues. The transcription levels of SiHDZO1 and SiHDZO7 were relatively higher in root and capsule, but low in stem, flower and leaf. Additionally, SiHDZ21 showed specific expression in seed, whereas low in other tissues. These findings 
A

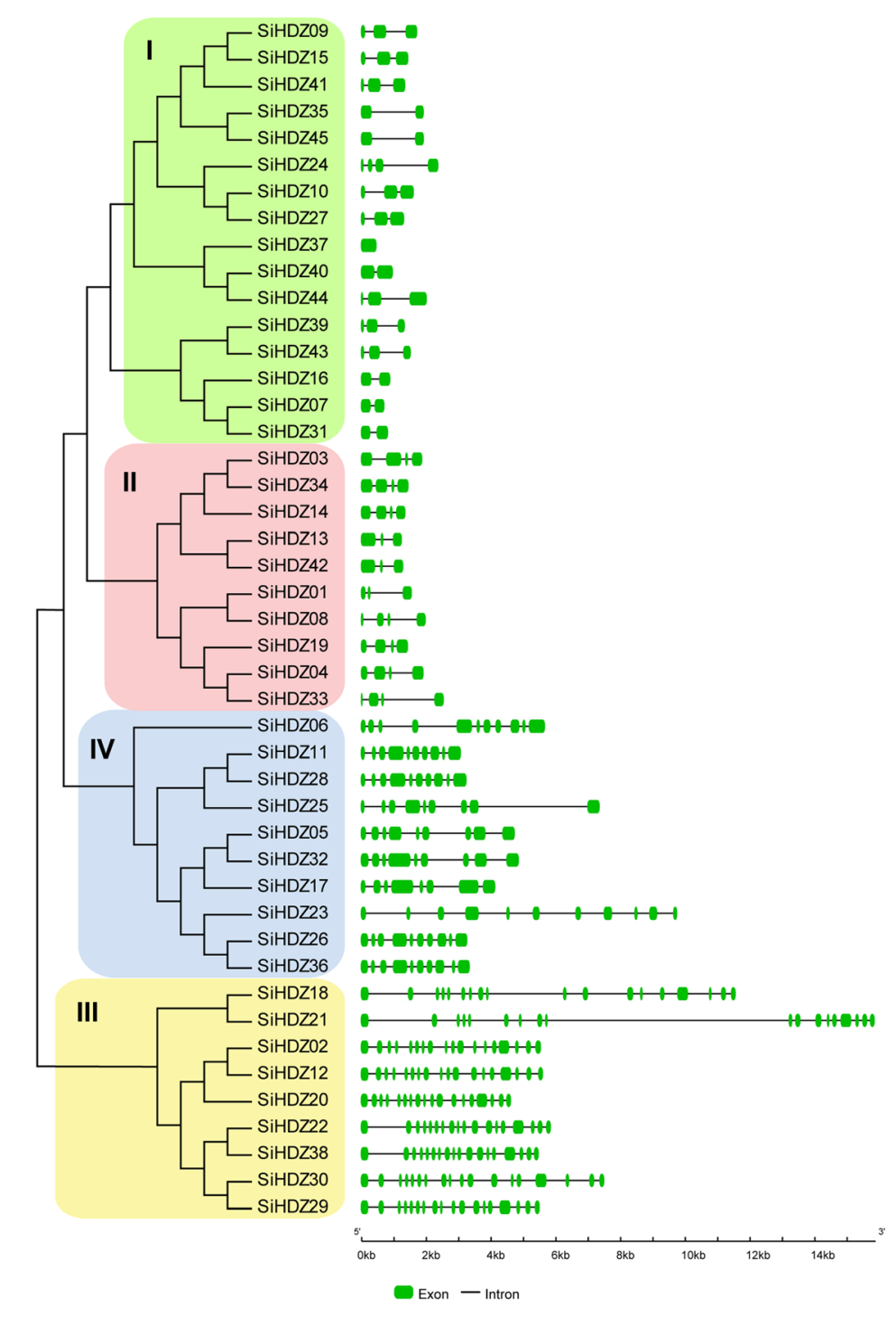

C

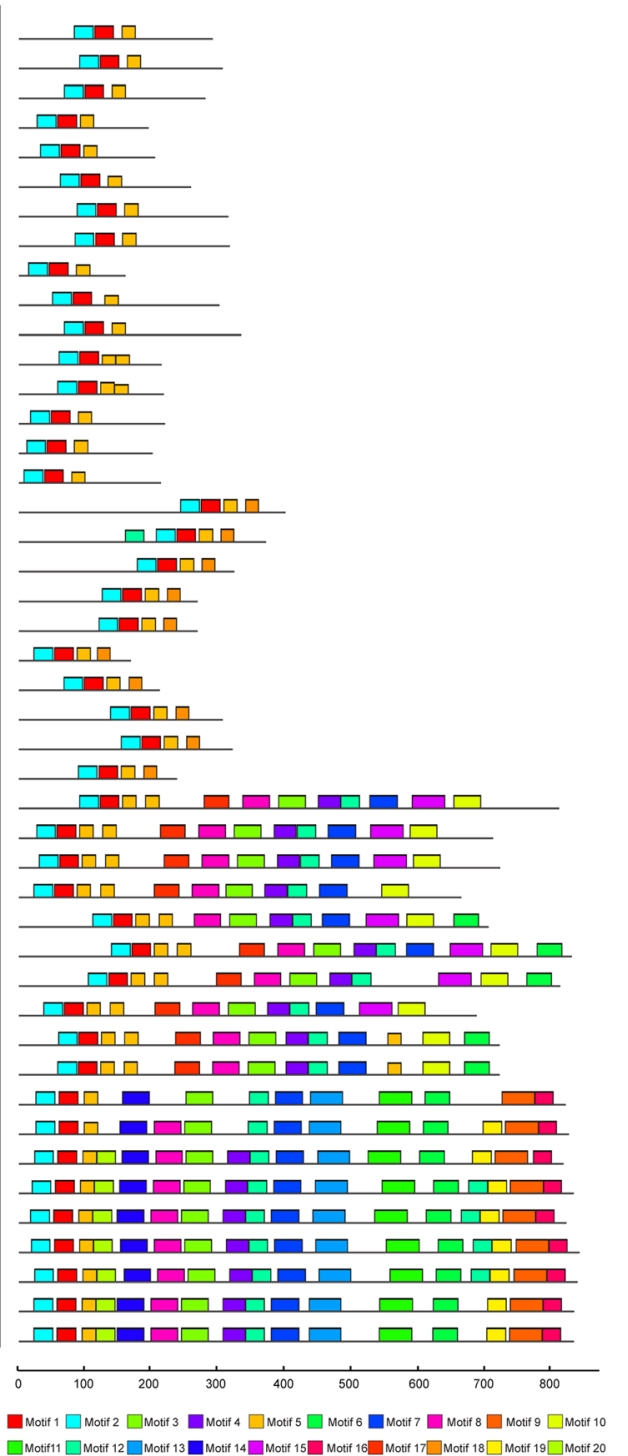

Fig. 3 The exon-intron structure and conserved domain organization of SiHDZ genes. a Phylogenetic relationships of SiHDZ genes. The SiHDZ amino acid sequences were aligned by Clustal $X$ and the tree was generated using the NJ method in MEGA 5.0 with 1000 bootstrap replicates. $\mathbf{b}$ exon-intron structures of SiHDZ genes. Exons and introns are shown as green boxes and black lines, respectively. c The conserved domain analysis of SiHDZ proteins using MEME. Different colors represent the different conserved motifs. The motif sequences are presented in Additional file 1: Figure $\mathbf{S 2}$

indicated the $\operatorname{SiHDZ}$ genes play differential roles in tissue development.

\section{Expression profiles of SiHDZ genes under drought stress}

To identify the expression profiles of SiHDZ genes under drought stress, the expression pattern of each $S i H D Z$ gene was obtained from the transcriptome data sets previously developed by our group [50]. It was observed that most of the $\mathrm{SiHDZ}$ genes were differentially affected under drought stress. For example, three SiHDZ genes, SiHDZ16, SiHDZ27, and SiHDZ31, were up-regulated under drought stress, with their expression markedly increasing at $3 \mathrm{~d}$, and peaking at $11 \mathrm{~d}$ (Fig. 5). Some SiHDZ genes, such as SiHDZ24, SiHDZO3, and SiHDZ42, were also up-regulated under stress, but their expression levels were peaked at $3 \mathrm{~d}$, implying their roles in early response to drought stress. However, some SiHDZ genes, such as SiHDZ30, SiHDZ14, SiHDZ22, and SiHDZ37, showed obvious down-regulation at all of the time points (Fig. 5). Moreover, some highly homologous $\mathrm{SiHDZ}$ genes exhibited similar expression profiles after drought stress, such as 


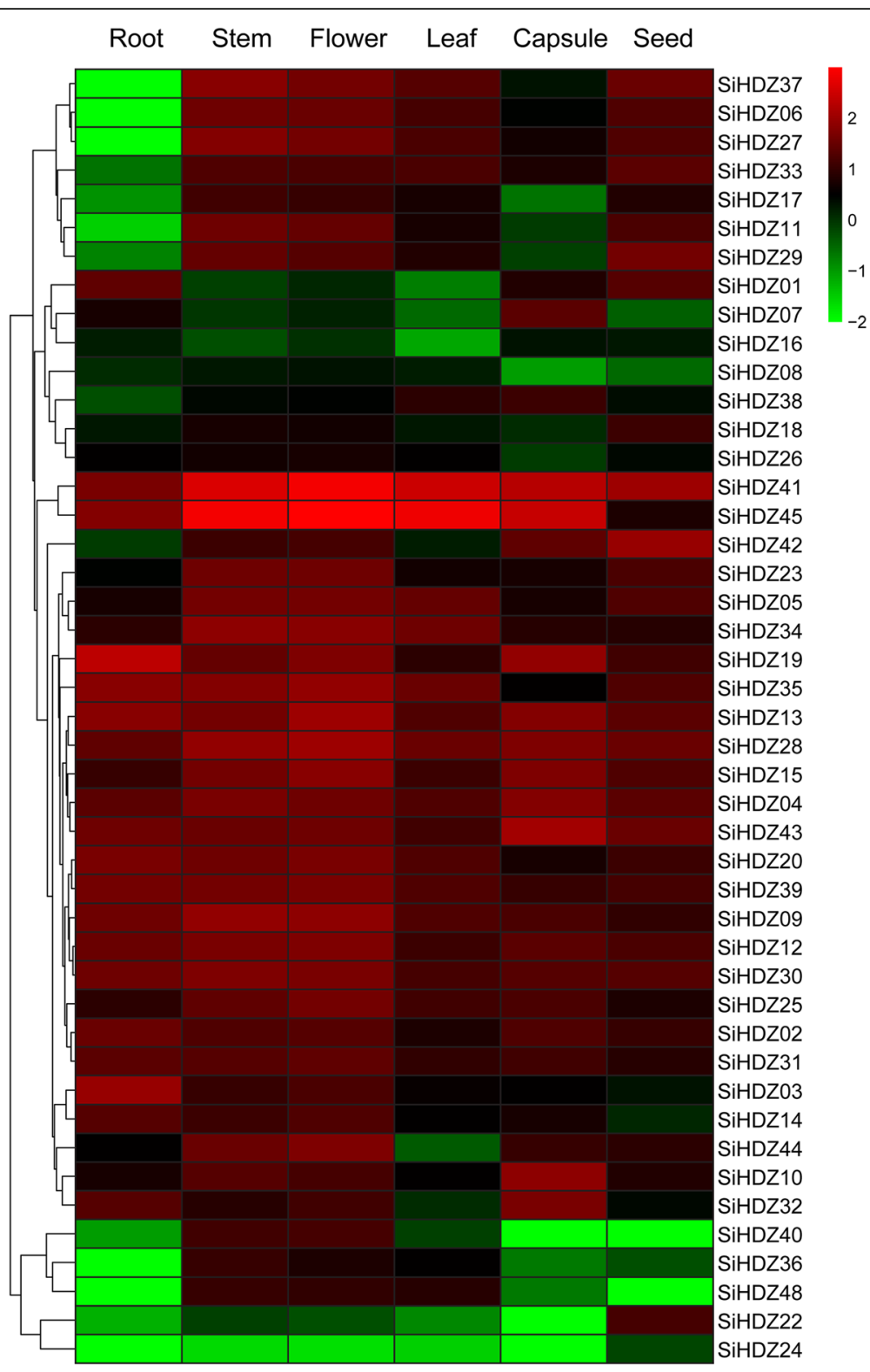

Fig. 4 Cluster analysis of the SiHDZs expression profiles in root, stem, flower, leaf, capsule and seed. The heatmap shows the log 10-transformed TPM values of each gene. The expression level is represented using color scale ranging from green (low expression) to red (high expression)

SiHDZ16 and SiHDZ31, SiHDZ19 and SiHDZ04, SiHDZ12 and SiHDZO2 (Fig. 5).

\section{Expression analysis of SiHDZ genes in response to osmotic and salinity stresses}

Numerous evidences showed that HD-Zip genes play a critical role in plant drought and salt tolerance [28, 51-53]. In order to identify candidate $\mathrm{SiHDZ}$ genes that function in drought and salt stress responses, 20 drought-responsive SiHDZs (based on the transcriptome data) from different subfamilies were selected to analyze their expression patterns under osmotic and salinity stresses by qRT-PCR. Overall, the expression levels of all the selected genes were significantly changed in response to osmotic and salinity stresses, but some differences were present among these genes. Under osmotic stress, the expression levels of SiHDZ03, 07, 10, 13, 16, 24, 27, 31, 34, and 43 were significantly up-regulated through all the time points, while SiHDZ22, 29, 41, and 42 were induced at certain time points, with the highest expression levels at $6 \mathrm{~h}, 6 \mathrm{~h}, 12 \mathrm{~h}$, and $12 \mathrm{~h}$, respectively (Fig. 6 and Additional file 1: Figure S3). However, other SiHDZ genes displayed an obvious decrease in expression under osmotic stress at certain time points. Under salinity stress, half of the selected $S i H D Z$ genes (SiHDZO3, 07, 10, 13, 16, 24, 27, 34, 42, and 43) showed strong up-regulation in expression at all of the 


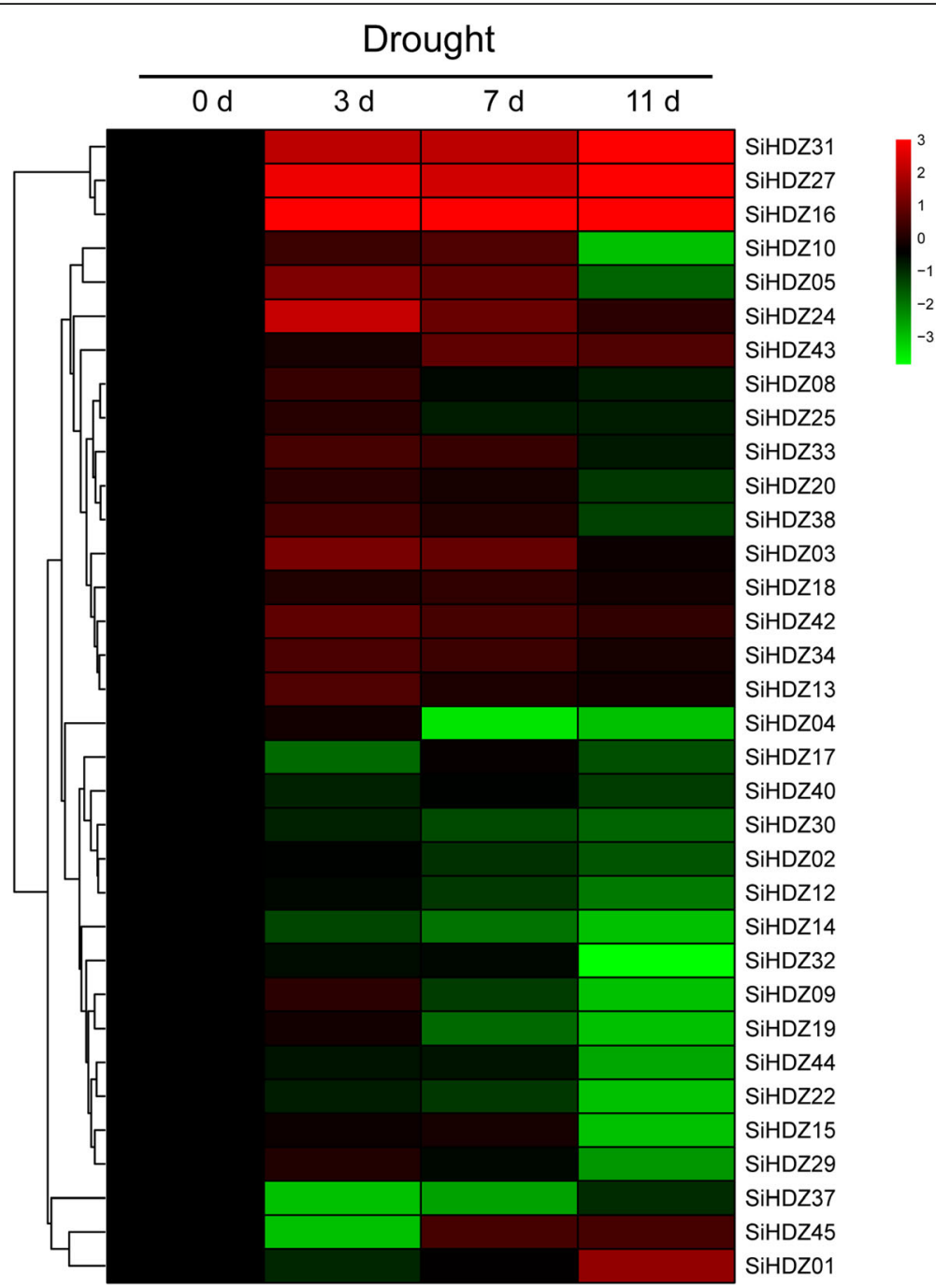

Fig. 5 Cluster analysis of the SiHDZs expression profiles under drought stress. The heatmap was created based on the log2-transformed values of the relative expression levels of the SiHDZ genes under drought stress in drought-tolerant accession ZZM0635. The expression level is represented using color scale ranging from green (down-regulated) to red (up-regulated)

time points, while SiHDZ26, 28, 29, 33, 37, and $41 \mathrm{kept}$ low transcription levels in some time points (Fig. 7). In addition, SiHDZ26, 29, 31, and 37 showed obviously increased expression at $2 \mathrm{~h}$ under salinity stress, while the transcripts were dramatically decreased at $6 \mathrm{~h}$ and $12 \mathrm{~h}$ (Fig. 7). Taken together, we found that the expression of several SiHDZ genes, such as SiHDZ07, 10, 27 and 43 was highly induced under both osmotic and salt treatments. These results suggested that these genes might play a vital role in response to multiple abiotic stresses in sesame.

\section{Discussion}

HD-Zip genes encode a family of plant-specific transcription factors involved in various biological processes in plants. In this study, a total of $45 \mathrm{SiHDZ}$ genes were identified from the sesame genome (Additional file 2: Table
S1). The number of HDZ members in sesame was comparable to that of potato (43) [23], wheat (46) [21], foxtail millet (Setaria italica) (47) [54], rice (48) [14, 15], and Arabidopsis (48) [6], more than tea plant (33) [22], but less than maize (55) [17] and cassava (57) [20]. These findings suggested that the number of HD-Zip genes is not correlated with genome size of these plant species, which may partly result from tandem duplication and segmental duplication. For instance, over 75\% of Arabidopsis HD-Zip genes are evolved from segmental duplication and are not clustered on the chromosomes $[17,55]$. In cassava, ten HD-Zip genes were identified as segmentally duplicated, while none of the HD-Zip genes were resulted from tandem duplication [20]. In the current study, $36 \mathrm{SiHDZ}$ genes were found to be involved in segmental duplication events, whereas no tandem duplicated $\mathrm{SiHDZ}$ genes were 

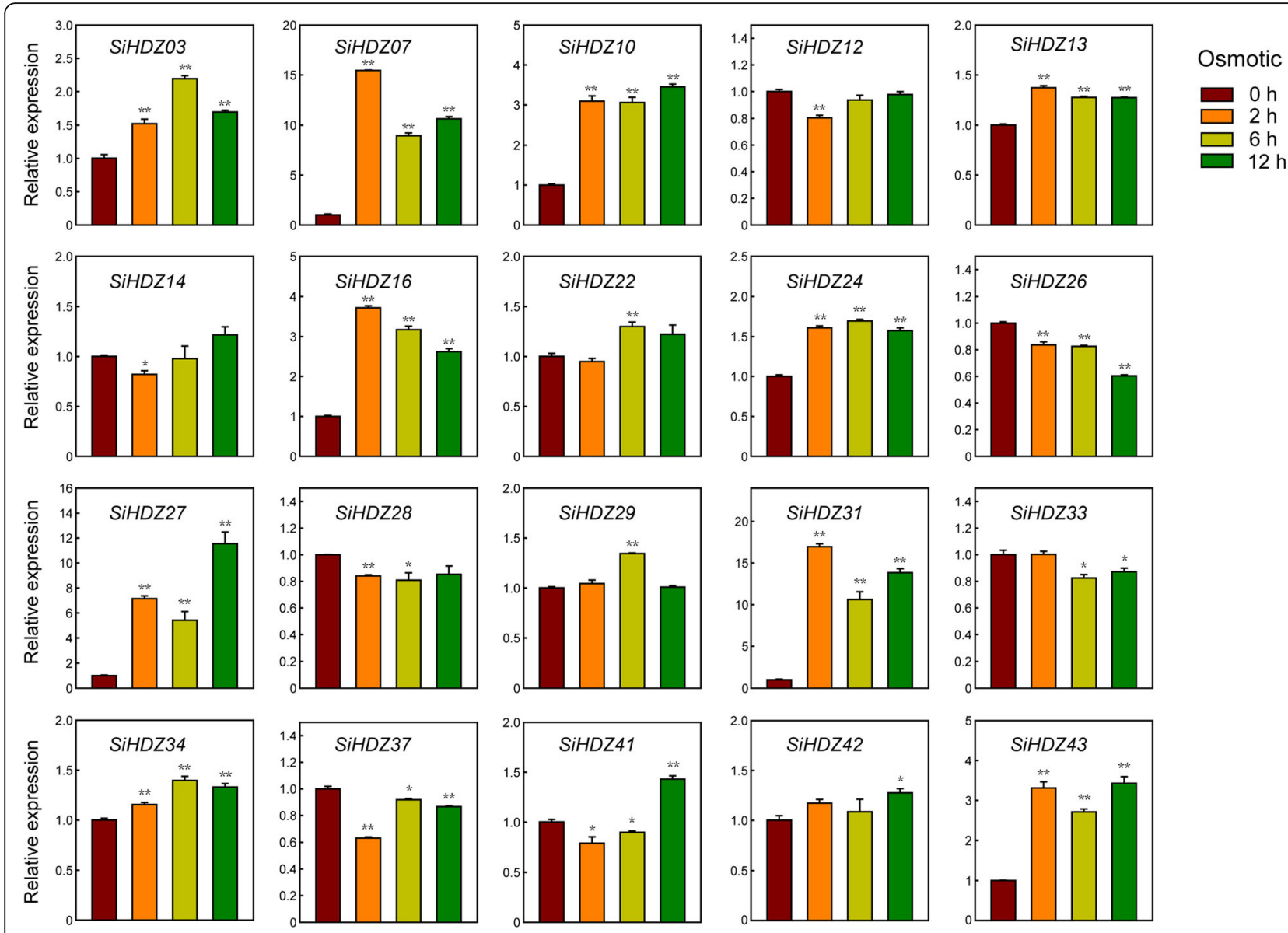

Fig. 6 Expression patterns of 20 SiHDZ genes under osmotic stress. Two-week-old seedlings were subjected to osmotic (15\% PEG 6000) stresses. Relative expression levels of SiHDZ genes were analyzed by qRT-PCR, using sesame SiH3.3 gene as the internal control. Error bars indicate standard deviations (SD) based on three replicates. ${ }^{*} P<0.05 ;{ }^{*} P<0.01, t$ test

identified, indicating that the segmental duplication was largely responsible for the expansion of HD-Zip gene family in sesame, which is consistent with the results in Arabidopsis, cassava and other species $[17,18,20]$.

The phylogenetic tree constructed using HDZ proteins from sesame and Arabidopsis revealed that the sesame HDZ proteins can be classified into four groups (HD-Zip I-IV) (Fig. 2). In addition, the number of HD-Zip I, II, III, and IV was 16, 10, 9, and 10, respectively (Additional file 2: Table S1; Fig. 2), compared to 17, 10, 5, and 16 in Arabidopsis [6], 11, 7, 5, and 8 in grape [16], 17, 18, 5, and 15 in maize [17], and 20, 17, 4, and 5 in wheat [21]. These results revealed that HD-Zip I was the most abundant group, but the number of HD-Zip III proteins in sesame was much higher than those in other plant species, accounting for $20 \%$. Noteworthy, 7 out of 9 SiHDZ genes in HD-Zip III subfamily were found to be involved in segmental duplication events (Fig. 2; Additional file 1: Figure S1), suggesting that the HD-Zip III SiHDZ genes also mainly expanded during evolution and may play important roles in sesame. Besides, the number of introns in
SiHDZ family genes varied widely (0-17), but members of the same group have similar exon-intron arrangements (Fig. 3a, b). For example, most SiHDZ genes in group III and IV harbored 17 and 8-10 introns, respectively, while members in group I and II had the least number of introns (1-3) (Fig. 3b), which is in accordance with the results in other plant species, such as maize [17], cassava [20], and potato [23]. The differences in the gene structures of different groups suggested group-specific gain or loss of introns that may affect the functional divergence of the different SiHDZ group members in sesame. The motif analysis also showed that the distribution of protein motifs in the different groups was noticeably diverse, but the same group had a similar motif construction (Fig. 3a, c), which support their strong evolutionary conservation. These findings suggested that the classification and evolution of the HD-Zip gene family is quite conserved in the sesame, as well as in other plant species.

Increasing evidences have demonstrated that HD-Zip genes participate in various aspects of growth and development in plants $[2,30,31,56]$. For example, sunflower 

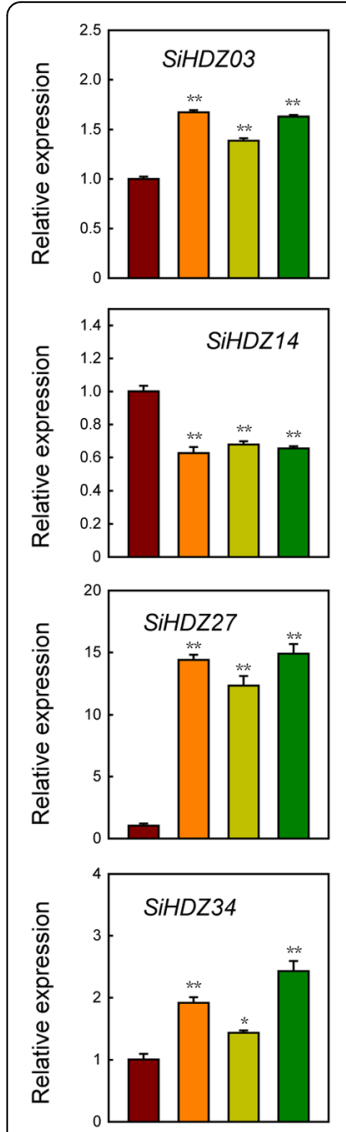
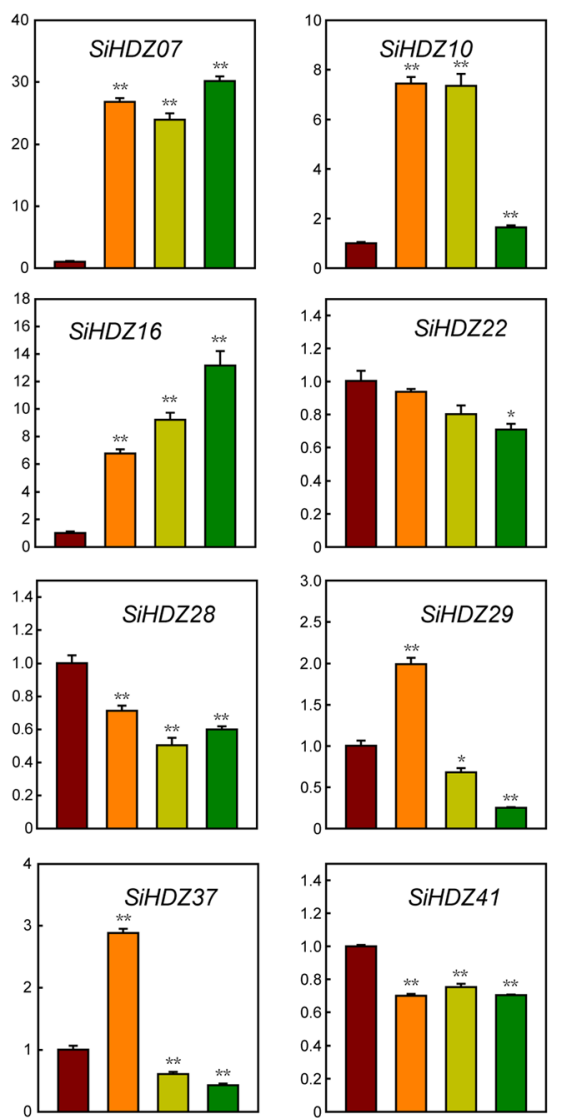
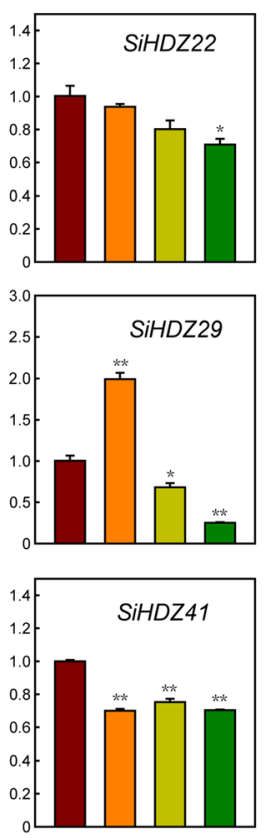
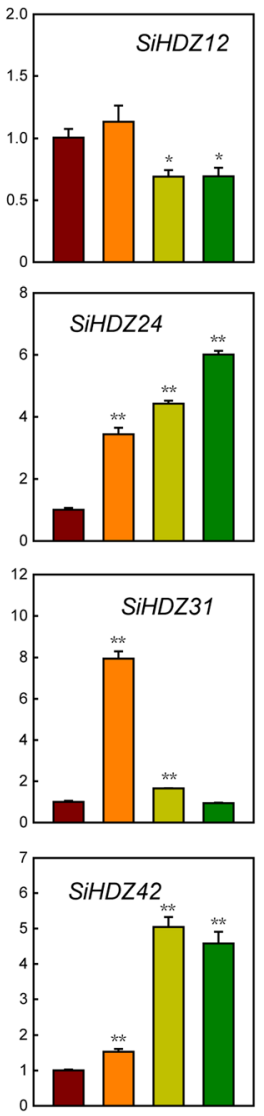
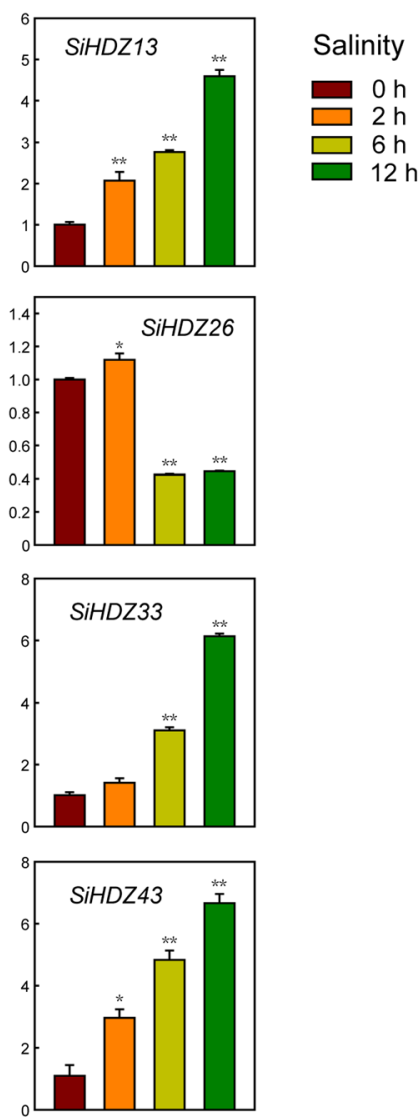
expression levels of SiHDZ genes were analyzed by qRT-PCR, using sesame SiH3.3 gene as the internal control. Error bars indicate standard deviations (SD) based on three replicates. ${ }^{*} P<0.05$; ${ }^{*} P<0.01, t$ test

HaHB10 (Helianthus ANNUUS HOMEOBOX 10) mediates the transition from the vegetative to the flowering stage by activating particular flowering transition genes and plant response to salicylic acid [57]. AtHB2 regulates $\mathrm{red} /$ far-red light effects on the shade avoidance response and specific cell proliferation including lateral root formation [58]. SiHDZ19 is the homologous gene of AtHB2 (Fig. 2), and was highly expressed in the root (Fig. 4), suggesting that it may also be involved in root development in sesame. It's worth noting that some SiHDZ genes, especially in HD-Zip I, II, and III, have a broad expression in all tissues (Fig. 4), implying that they participate in the overall development of the sesame. Similar results were also observed in several plant species, such as wheat [21], and potato [23]. However, most HDZip IV SiHDZ genes displayed distinct tissue-specific expression patterns (Fig. 4), similar to the Group IV genes in cassava [20]. For example, SiHDZ06, 11, 17, 26, 28, and 36 have a much lower expression in roots than in other tissues, while another HD-Zip IV gene, SiHDZ23, was specifically expressed in seed (Fig. 4). In cucumber, nearly all HD-Zip IV genes (10 out of 11) showed preferential expression in reproductive organs [59]. Tomato HD-Zip IV genes also showed tissue specific expression patterns, with higher expression in young leaves and flowers [60]. These findings revealed that the functions of different group $\mathrm{SiHDZ}$ genes were diverged during evolution processes.

Numerous reports have revealed that HD-Zip genes were involved in response to a variety of abiotic stresses in different plant species, such as foxtail millet [54], tea plant [22], wheat [21], and potato [23]. In the present study, the expression levels of over 75\% SiHDZ genes were found to be affected by drought or salinity stresses based on transcriptome data and qRT-PCR results. Moreover, SiHDZ03, 07, 10, 13, 16, 24, 27, 31, and 43, have similar expression patterns in response to drought and salinity. A large body of evidence indicates that HDZip I proteins are involved in developmental reprogramming in response to environmental stresses [26]. For example, AtHB7 and $A t H B 12$, which are strongly induced by water limiting and $\mathrm{ABA}$, act as mediators that negatively feedback effect on ABA signaling in the plant response to water deficit [26, 27]. The ectopically 
expression and/or overexpression of the Arabidopsis AtHB12 or AtHB7 gene, or their homologs in M. truncatula (MtHB1), sunflower (HaHB4), and maize (ZmHDZ4), enhanced tolerance to drought or salinity stress in transgenic plants [29, 61-63]. Some of the HDZip I genes in sesame, such as SiHDZO7, 10, 16, 24, 27, 31,37 , and 43 , were significantly induced in response to drought or salt stress. Notably, the expression of SiHDZ07, 16, and 31, homologs of Arabidopsis AtHB12 and $A t H B 7$ genes, was significantly up-regulated by both drought and salinity stresses, indicating that these genes may regulate drought and salt tolerance through an ABA-dependent pathway. In addition, SiHDZ43, like its homologs $A t H B 21$ and $A t H B 40$, was up-regulated under drought and salinity stress. Three HD-Zip II members, SiHDZ03, 13, and 42, were also significantly upregulated under drought and/or salt treatment, thereby suggesting their possible role in abiotic stress responses. These results suggest HD-Zip I and II genes may play a vital role in regulating plant tolerance to the adverse environmental conditions.

\section{Conclusions}

In this study, we identified $45 \mathrm{HD}$-Zip genes from sesame at the whole genome level. Phylogenetic analysis identified four subfamilies (HD-Zip I-IV) in the HD-Zip gene family, which was further supported by the analysis of their conserved motifs and gene structures. Transcriptomic analysis revealed some constitutively or tissue-specific expressed HD-Zip genes. Expression profiles of SiHDZ genes under various abiotic stress treatments indicated that over $75 \%$ SiHDZ genes are involved in abiotic stress signaling, and members of HD-Zip I and II subfamilies may play a vital role in regulating plant response to abiotic stresses. Together, these data provide useful information for functional characterization of SiHDZ genes and extend our knowledge of abiotic stress response in sesame.

\section{Methods}

\section{Genome-wide identification of HD-zip family genes in sesame}

To identify HD-Zip protein in sesame, all proteins sequences were downloaded from the Sesamum indicum genome database (Sinbase, http://ocri-genomics.org/ Sinbase/index.html) [64]. A local protein database was constructed and searched against known HD-Zip protein sequences collected from Arabidopsis using a local protein basic local alignment search (BLASTP) program with an E-value cut-off $<10-5$ and an identity of $50 \%$ as the threshold. HMM profile of the homeodomain (PF00046) and the leucine zipper domain (PF02183) were download from the PFAM database and used for local Hidden Markov Model (HMM) search by HMMER3.0 [65]. Subsequently, all obtained protein sequences were further examined by SMART (http://smart.embl-heidelberg.de/) to confirm the presence of the HD and LZ domains. Finally, a total of 45 non-redundant sesame HDZip encoding genes were identified.

\section{Phylogenetic analysis and gene duplication}

The protein sequences of HD-Zip from sesame and were Arabidopsis used to construct the phylogenetic tree by MEGA 5.2, using the neighbor-joining (NJ) method with 1000 bootstrap replications [66]. Gene duplication was analyzed with MCScanX following the method described previously [67].

\section{Gene structure and protein conserved motifs analysis}

The exon/intron organization of the HD-Zip genes in sesame was performed with Gene Structure Display Server (GSDS) (http://gsds.cbi.pku.edu.cn/index.php). Conserved motifs present in SiHDZs were identified using MEME (Multiple Em for Motif Elicitation) v4.11.4 (http://meme-suite.org/tools/meme).

\section{Expression profiling of SiHDZ genes using available transcriptome data}

To gain insight into the tissue-specific gene expression patterns of HD-Zip gene, transcriptome data for six tissues (root, stem, flower, leaf, capsule and seed) were extracted from Sesame Functional Genomics Database (SesameFG, http://www.ncgr.ac.cn/SesameFG) [49]. To analysis the expression profiles of SiHDZ genes in response to drought stress, the transcriptome data (accession number SAMN06130606) were used [50]. The hierarchical cluster analyses of gene expression were performed using Cluster 3.0 software [68], and heatmaps were visualized with TreeView [69].

\section{Plant materials and treatments}

In this study, Two-week old seedlings of Sesame cultivar Zhongzhi No. 13 were used to examine the expression patterns of SiHDZ genes under osmotic and salt stresses. Plants were grown in a growth chamber at $28 \pm 2{ }^{\circ} \mathrm{C}$ with a 16-h light/8-h dark photoperiod, and exposed to different stresses as described previously [70]. The shoots tissues were collected at $0,2,6$ and $12 \mathrm{~h}$ after treatment. Three biological replications were conducted per sample.

\section{Quantitative real-time RT-PCR}

Total RNA isolation and the first-strand cDNA synthetization were performed as described previously [70]. The gene-specific primers used in this study are listed in Additional file 3: Table S2. Quantitative real-time RT-PCR (qRT-PCR) was performed according to the protocol described previously [70]. Significant up- and down-regulated genes were determined as $p<0.05$ or $p<0.01$ using $t$ test. 


\section{Supplementary information}

Supplementary information accompanies this paper at https://doi.org/10. 1186/s12864-019-6091-5

Additional file 1: Fig. S1. Segmental duplicated SiHDZ genes on 16 linkage groups. Red lines indicate duplicated SiHDZ gene pairs. Grey lines indicate collinear blocks in whole sesame genome. Fig. S2. The logos of 20 conserved motifs in SiHDZ proteins. Fig. S3. Expression profiles of stress marker genes under osmotic and salinity stress treatments.

Additional file 2. Table S1. HD-ZIP transcription factor gene family in sesame.

Additional file 3. Table S2. List of primers used for quantitative realtime RT-PCR analysis.

\section{Abbreviations}

AtHB: Arabidopsis thaliana homeobox; HD: Homeodomain; HD-

Zip: Homeodomain-leucine zipper; HMM: Hidden Markov model; qRT-

PCR: Quantitative real-time reverse transcription-polymerase chain reaction; SiHDZ: Sesamum indicum homeodomain-leucine zipper; TF: Transcription factor

\section{Acknowledgments}

Not applicable.

\section{Authors' contributions}

$\mathrm{XZ}$ and JY conceived and designed the experiments; MW and AL performed the experiments; MW, AL, YZha, YZho, DL, KD, and RZ participated in data collection and analysis; MW, KD, and JY wrote the paper. All authors have read and approved the final manuscript.

\section{Funding}

This research was funded by China Agriculture Research System (CARS-14), the Agricultural Science and Technology Innovation Project of Chinese Academy of Agricultural Sciences (CAAS-ASTIP-2016-OCRI), and Central Public-interest Scientific Institution Basal Research Fund (1610172018007). The funders had no role in the experimental design, data collection and analysis or writing the manuscript.

\section{Availability of data and materials}

Gene sequence information of HD-Zip in sesame is available at the Sesamum indicum genome database (Sinbase, http://ocri-genomics.org/Sinbase/index. html).

\section{Ethics approval and consent to participate}

Not applicable.

\section{Consent for publication}

Not applicable.

\section{Competing interests}

The authors declare that they have no competing interests.

\section{Author details}

${ }^{1}$ Key Laboratory of Biology and Genetic Improvement of Oil Crops, Ministry of Agriculture and Rural Affairs, Oil Crops Research Institute of the Chinese Academy of Agricultural Sciences, Wuhan 430062, China. ${ }^{2}$ Special Economic Crop Research Center of Shandon Academy of Agricultural Sciences, Shandong Cotton Research Center, Jinan 250100, China. ${ }^{3}$ Key Laboratory of Crop Physiology, Ecology and Genetic Breeding, Ministry of Education, Jiangxi Agricultural University, Nanchang 330045, China.

Received: 5 May 2019 Accepted: 10 September 2019 Published online: 16 October 2019

\section{References}

1. Inukai S, Kock KH, Bulyk ML. Transcription factor-DNA binding: beyond binding site motifs. Curr Opin Genet Dev. 2017;43:110-9.

2. Ariel FD, Manavella PA, Dezar CA, Chan RL. The true story of the HD-zip family. Trends Plant Sci. 2007;12(9):419-26.
3. Kissinger CR, Liu BS, Martin-Blanco E, Kornberg TB, Pabo CO. Crystal structure of an engrailed homeodomain-DNA complex at 2.8 a resolution: a framework for understanding homeodomain-DNA interactions. Cell. 1990:63(3):579-90.

4. Ottinng G, Qian YQ, Billeter M, Muller M, Affolter M, Gehring WJ, Wuthrich K Protein-DNA contacts in the structure of a homeodomain-DNA complex determined by nuclear magnetic resonance spectroscopy in solution. EMBO J. 1990;9(10):3085-92.

5. Gehring WJ, Qian YQ, Billeter M, Furukubo-Tokunaga K, Schier AF, ResendezPerez D, Affolter M, Otting G, Wüthrich K. Homeodomain-DNA recognition. Cell. 1994:78(2):211-23.

6. Mukherjee K, Brocchieri L, Burglin TR. A comprehensive classification and evolutionary analysis of plant homeobox genes. Mol Biol Evol. 2009;26(12):2775-94

7. Sessa G, Morelli G, Ruberti I. The Athb-1 and -2 HD-zip domains homodimerize forming complexes of different DNA binding specificities. EMBO J. 1993:12(9):3507-17.

8. Tron AE. Combinatorial interactions of two amino acids with a single base pair define target site specificity in plant dimeric homeodomain proteins. Nucleic Acids Res. 2001;29(23):4866-72.

9. Chan RL, Gago GM, Palena CM, Gonzalez DH. Homeoboxes in plant development. Biochim Biophys Acta. 1998;1442(1):1-19.

10. Schrick K, Nguyen D, Karlowski WM, Mayer KF. START lipid/sterol-binding domains are amplified in plants and are predominantly associated with homeodomain transcription factors. Genome Biol. 2004;5(6):R41.

11. Mukherjee K, Burglin TR. MEKHLA, a novel domain with similarity to PAS domains, is fused to plant homeodomain-leucine zipper III proteins. Plant Physiol. 2006;140(4):1142-50.

12. Sessa G, Steindler C, Morelli G, Ruberti I. The Arabidopsis Athb-8, -9 and -14 genes are members of a small gene family coding for highly related HD-ZIP proteins. Plant Mol Biol. 1998;38(4):609-22.

13. Abe M, Takahashi T, Komeda Y. Identification of a cis-regulatory element for L1 layer-specific gene expression, which is targeted by an L1-specific homeodomain protein. Plant J. 2001:26(5):487-94.

14. Jain M, Tyagi AK, Khurana JP. Genome-wide identification, classification, evolutionary expansion and expression analyses of homeobox genes in rice. FEBS J. 2008;275(11):2845-61.

15. Agalou A, Purwantomo S, Overnas E, Johannesson H, Zhu X, Estiati A, de Kam RJ, Engstrom P, Slamet-Loedin IH, Zhu Z, et al. A genome-wide survey of HD-zip genes in rice and analysis of drought-responsive family members. Plant Mol Biol. 2008;66(1-2):87-103.

16. Jiang $H$, Jin J, Liu H, Dong Q, Yan H, Gan D, Zhang W, Zhu S. Genome-wide analysis of HD-zip genes in grape (Vitis vinifera). Tree Genet Genomes. 2015;11(1):827.

17. Lespinet O, Zhao Y, Zhou Y, Jiang H, Li X, Gan D, Peng X, Zhu S, Cheng B. Systematic analysis of sequences and expression patterns of droughtresponsive members of the HD-zip gene family in maize. PLoS One. 2011;6(12):e28488

18. Ali J, Chen X, Chen Z, Zhao H, Zhao Y, Cheng B, Xiang Y. Genome-wide analysis of soybean HD-zip gene family and expression profiling under salinity and drought treatments. PLoS One. 2014:9(2):e87156.

19. Belamkar V, Weeks NT, Bharti AK, Farmer AD, Graham MA, Cannon SB. Comprehensive characterization and RNA-Seq profiling of the HD-zip transcription factor family in soybean (Glycine max) during dehydration and salt stress. BMC Genomics. 2014:15(1):950.

20. Ding Z, Fu L, Yan Y, Tie W, Xia Z, Wang W, Peng M, Hu W, Zhang J. Genome-wide characterization and expression profiling of HD-zip gene family related to abiotic stress in cassava. PLoS One. 2017;12(3):e0173043.

21. Yue $H$, Shu D, Wang $M$, Xing G, Zhan H, Du X, Song W, Nie X. Genomewide identification and expression analysis of the HD-zip gene family in wheat (Triticum aestivum L.). Genes (Basel). 2018;9(2):70.

22. Shen W, Li H, Teng R, Wang Y, Wang W, Zhuang J. Genomic and transcriptomic analyses of HD-zip family transcription factors and their responses to abiotic stress in tea plant (Camellia sinensis). Genomics. 2018; 111:1142-1151.

23. Li W, Dong J, Cao M, Gao X, Wang D, Liu B, Chen O. Genome-wide identification and characterization of HD-ZIP genes in potato. Gene. 2019:697:103-17.

24. Sessa G, Carabelli M, Possenti M, Morelli G, Ruberti I. Multiple links between HD-Zip proteins and hormone networks. Int J Mol Sci. 2018; 19(12):4047. 
25. Roodbarkelari F, Groot EP. Regulatory function of homeodomain-leucine zipper (HD-ZIP) family proteins during embryogenesis. New Phytol. 2017; 213(1):95-104.

26. Gong S, Ding Y, Hu S, Ding L, Chen Z, Zhu C. The role of HD-zip class I transcription factors in plant response to abiotic stresses. Physiol Plant. 2019 https://doi.org/10.1111/ppl.12965.

27. Valdes $A E$, Overnas E, Johansson H, Rada-Iglesias A, Engstrom P. The homeodomain-leucine zipper (HD-zip) class I transcription factors ATHB7 and $\mathrm{ATHB} 12$ modulate abscisic acid signalling by regulating protein phosphatase $2 \mathrm{C}$ and abscisic acid receptor gene activities. Plant Mol Biol. 2012;80(4-5):405-18.

28. Re DA, Capella M, Bonaventure G, Chan RL. Arabidopsis AtHB7 and AtHB12 evolved divergently to fine tune processes associated with growth and responses to water stress. BMC Plant Biol. 2014;14:150.

29. Ariel F, Diet A, Verdenaud M, Gruber V, Frugier F, Chan R, Crespi M. Environmental regulation of lateral root emergence in Medicago truncatula requires the HD-zip I transcription factor HB1. Plant Cell. 2010;22(7):2171-83.

30. Turchi L, Carabelli M, Ruzza V, Possenti M, Sassi M, Penalosa A, Sessa G, Salvi S, Forte V, Morelli G, et al. Arabidopsis HD-zip II transcription factors control apical embryo development and meristem function. Development. 2013; 140(10):2118-29.

31. Turchi L, Baima S, Morelli G, Ruberti I. Interplay of HD-zip II and III transcription factors in auxin-regulated plant development. J Exp Bot. 2015; 66(16):5043-53.

32. Ciarbelli AR, Ciolfi A, Salvucci S, Ruzza V, Possenti M, Carabelli M, Fruscalzo A Sessa G, Morelli G, Ruberti I. The Arabidopsis homeodomain-leucine zipper II gene family: diversity and redundancy. Plant Mol Biol. 2008;68(4-5):465-78.

33. Carabelli M, Possenti M, Sessa G, Ruzza V, Morelli G, Ruberti I. Arabidopsis HD-zip II proteins regulate the exit from proliferation during leaf development in canopy shade. J Exp Bot. 2018;69(22):5419-31.

34. Park MY, Kim SA, Lee SJ, Kim SY. ATHB17 is a positive regulator of abscisic acid response during early seedling growth. Mol Cells. 2013;35(2):125-33.

35. Liu T, Longhurst AD, Talavera-Rauh F, Hokin SA, Barton MK. The Arabidopsis transcription factor $A B I G 1$ relays $A B A$ signaled growth inhibition and drought induced senescence. Elife. 2016;5:e13768.

36. Ramachandran P, Carlsbecker A, Etchells JP. Class III HD-ZIPs govern vascular cel fate: an HD view on patterning and differentiation. J Exp Bot. 2017;68(1):55-69.

37. Zhang T, Li Y, Ma L, Sang X, Ling Y, Wang Y, Yu P, Zhuang H, Huang J, Wang $N$, et al. LATERAL FLORET 1 induced the three-florets spikelet in rice. Proc Natl Acad Sci U S A. 2017;114(37):9984-9.

38. Zhang F, Zuo K, Zhang J, Liu X, Zhang L, Sun X, Tang K. An L1 box binding protein, GbML1, interacts with GbMYB25 to control cotton fibre development. J Exp Bot. 2010;61(13):3599-613.

39. Shan CM, Shangguan XX, Zhao B, Zhang XF, Chao LM, Yang CQ, Wang L, Zhu HY, Zeng YD, Guo WZ, et al. Control of cotton fibre elongation by a homeodomain transcription factor GhHOX3. Nat Commun. 2014;5:5519.

40. Makinde FM, Akinoso R. Comparison between the nutritional quality of flour obtained from raw, roasted and fermented sesame (Sesamum indicum L.) seed grown in Nigeria. Acta Sci Pol Technol Aliment. 2014;13(3):309-19.

41. Dossa K, Diouf D, Wang L, Wei X, Zhang Y, Niang M, Fonceka D, Yu J, Mmadi MA, Yehouessi LW, et al. The emerging oilseed crop Sesamum indicum enters the "omics" era. Front Plant Sci. 2017;8:1154.

42. Wei W, Li D, Wang L, Ding X, Zhang Y, Gao Y, Zhang X. Morpho-anatomical and physiological responses to waterlogging of sesame (Sesamum indicum L.). Plant Sci. 2013;208:102-11.

43. Hassanzadeh M, Asghari A, Jamaati-e-Somarin S, Saeidi M, Zabihi-eMahmoodabad R, Hokmalipour S. Effects of water deficit on drought tolerance indices of sesame (Sesamum indicum L.) genotypes in Moghan region. Res J Environ Sci. 2009;3:116-21.

44. Mmadi MA, Dossa K, Wang L, Zhou R, Wang Y, Cisse N, Sy MO, Zhang X. Functional characterization of the versatile MYB gene family uncovered their important roles in plant development and responses to drought and waterlogging in sesame. Genes (Basel). 2017:8(12):362.

45. Zhang Y, Li D, Wang Y, Zhou R, Wang L, Zhang Y, Yu J, Gong H, You $J$, Zhang $X$. Genome-wide identification and comprehensive analysis of the NAC transcription factor family in Sesamum indicum. PLoS One. 2018;13(6):e0199262

46. Wang Y, Zhang Y, Zhou R, Dossa K, Yu J, Li D, Liu A, Mmadi MA, Zhang X, You J. Identification and characterization of the bZIP transcription factor family and its expression in response to abiotic stresses in sesame. PLoS One. 2018;13(7):e0200850.
47. Dossa K, Wei X, Li D, Fonceka D, Zhang Y, Wang L, Yu J, Boshou L, Diouf D,

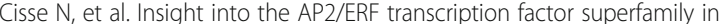
sesame and expression profiling of DREB subfamily under drought stress. BMC Plant Biol. 2016;16(1):171.

48. Li D, Liu P, Yu J, Wang L, Dossa K, Zhang Y, Zhou R, Wei X, Zhang X. Genome-wide analysis of WRKY gene family in the sesame genome and dentification of the WRKY genes involved in responses to abiotic stresses. BMC Plant Biol. 2017;17(1):152.

49. Wei X, Gong H, Yu J, Liu P, Wang L, Zhang Y, Zhang X. SesameFG: an integrated database for the functional genomics of sesame. Sci Rep. 2017;7(1):2342.

50. Dossa K, Li D, Wang L, Zheng X, Liu A, Yu J, Wei X, Zhou R, Fonceka D, Diouf $\mathrm{D}$, et al. Transcriptomic, biochemical and physio-anatomical investigations shed more light on responses to drought stress in two contrasting sesame genotypes. Sci Rep. 2017;7(1):8755.

51. Bang SW, Lee DK, Jung H, Chung PJ, Kim YS, Choi YD, Suh JW, Kim JK. Overexpression of OsTF1L, a rice HD-zip transcription factor, promotes lignin biosynthesis and stomatal closure that improves drought tolerance. Plant Biotechnol J. 2019;17(1):118-31.

52. Tan $W$, Zhang $D$, Zhou $H$, Zheng $T$, Yin $Y$, Lin $H$. Transcription factor HAT1 is a substrate of SnRK2.3 kinase and negatively regulates ABA synthesis and signaling in Arabidopsis responding to drought. PLoS Genet. 2018;14(4):e1007336.

53. Yang Y, Luang S, Harris J, Riboni M, Li Y, Bazanova N, Hrmova M, Haefele S, Kovalchuk N, Lopato S. Overexpression of the class I homeodomain transcription factor TaHDZipl-5 increases drought and frost tolerance in transgenic wheat. Plant Biotechnol J. 2018;16(6):1227-40.

54. Chai W, Si W, Ji W, Qin Q, Zhao M, Jiang H. Genome-wide investigation and expression profiling of HD-zip transcription factors in foxtail millet (Setaria italica L.). Biomed Res Int. 2018;2018:8457614.

55. Henriksson E, Olsson AS, Johannesson H, Johansson H, Hanson J, Engstrom $P$, Soderman E. Homeodomain leucine zipper class I genes in Arabidopsis. Expression patterns and phylogenetic relationships. Plant Physiol. 2005; 139(1):509-18.

56. Romani F, Reinheimer R, Florent SN, Bowman JL, Moreno JE. Evolutionary history of HOMEODOMAIN LEUCINE ZIPPER transcription factors during plant transition to land. New Phytol. 2018;219(1):408-21.

57. Dezar CA, Giacomelli Jl, Manavella PA, Re DA, Alves-Ferreira M, Baldwin IT, Bonaventure G, Chan RL. HAHB10, a sunflower HD-zip II transcription factor, participates in the induction of flowering and in the control of phytohormone-mediated responses to biotic stress. J Exp Bot. 2011; 62(3):1061-76.

58. Steindler C, Matteucci A, Sessa G, Weimar T, Ohgishi M, Aoyama T, Morelli G, Ruberti I. Shade avoidance responses are mediated by the ATHB-2 HD-zip protein, a negative regulator of gene expression. Development. 1999; 126(19):4235-45.

59. Fu R, Liu W, Li Q, Li J, Wang L, Ren Z. Comprehensive analysis of the homeodomain-leucine zipper IV transcription factor family in Cucumis sativus. Genome. 2013;56(7):395-405.

60. Gao Y, Gao S, Xiong C, Yu G, Chang J, Ye Z, Yang C. Comprehensive analysis and expression profile of the homeodomain leucine zipper IV transcription factor family in tomato. Plant Physiol Biochem. 2015;96:141-53.

61. Mishra KB, lannacone R, Petrozza A, Mishra A, Armentano N, La Vecchia G, Trtilek M, Cellini F, Nedbal L. Engineered drought tolerance in tomato plants is reflected in chlorophyll fluorescence emission. Plant Sci. 2012;182:79-86.

62. Romani F, Ribone PA, Capella M, Miguel VN, Chan RL. A matter of quantity: common features in the drought response of transgenic plants overexpressing HD-zip I transcription factors. Plant Sci. 2016;251:139-54.

63. Gonzalez FG, Capella M, Ribichich KF, Curin F, Giacomelli Jl, Ayala F, Watson G, Otegui ME, Chan RL. Field-grown transgenic wheat expressing the sunflower gene HaHB4 significantly outyields the wild type. J Exp Bot. 2019: 70(5):1669-81

64. Wang L, Yu J, Li D, Zhang X. Sinbase: an integrated database to study genomics, genetics and comparative genomics in Sesamum indicum. Plant Cell Physiol. 2015;56(1):e2.

65. Wheeler TJ, Eddy SR. Nhmmer: DNA homology search with profile HMMs. Bioinformatics. 2013;29(19):2487-9.

66. Tamura K, Peterson D, Peterson N, Stecher G, Nei M, Kumar S. MEGA5: molecular evolutionary genetics analysis using maximum likelihood, evolutionary distance, and maximum parsimony methods. Mol Biol Evol. 2011;28(10):2731-9. 
67. Wang Y, Tang H, Debarry JD, Tan X, Li J, Wang X, Lee TH, Jin H, Marler B, Guo H, et al. MCScanX: a toolkit for detection and evolutionary analysis of gene synteny and collinearity. Nucleic Acids Res. 2012;40(7):e49.

68. de Hoon MJ, Imoto S, Nolan J, Miyano S. Open source clustering software. Bioinformatics. 2004;20(9):1453-4.

69. Saldanha AJ. Java Treeview--extensible visualization of microarray data. Bioinformatics. 2004;20(17):3246-8.

70. You J, Wang Y, Zhang Y, Dossa K, Li D, Zhou R, Wang L, Zhang X. Genomewide identification and expression analyses of genes involved in raffinose accumulation in sesame. Sci Rep. 2018;8(1):4331.

\section{Publisher's Note}

Springer Nature remains neutral with regard to jurisdictional claims in published maps and institutional affiliations.

Ready to submit your research? Choose BMC and benefit from:

- fast, convenient online submission

- thorough peer review by experienced researchers in your field

- rapid publication on acceptance

- support for research data, including large and complex data types

- gold Open Access which fosters wider collaboration and increased citations

- maximum visibility for your research: over $100 \mathrm{M}$ website views per year

At $\mathrm{BMC}$, research is always in progress.

Learn more biomedcentral.com/submissions 